\title{
SIMULAÇÃO NUMÉRICA DOS FLUIDOS REFRIGERANTES R11, R12, R22, R134A, R410A E R717 APLICADOS NO CICLO DE REFRIGERAÇÃO POR COMPRESSÃO DE VAPOR CONVENCIONAL
}

\author{
J. P. dos REIS ${ }^{1}$ e W. SEIDEL ${ }^{1}$ \\ ${ }^{1}$ Católica de Santa Catarina em Jaraguá do Sul \\ E-mail para contato: jaissonreis@catolicasc.org.br
}

\begin{abstract}
RESUMO - O presente trabalho analisou um sistema de refrigeração convencional, tendo como objetivo geral realizar uma análise comparativa entre alguns fluidos refrigerantes (R11, R12, R22, R134A, R410A e R717) para um sistema de refrigeração convencional utilizando o software Engineering Equation Solver (EES). Analisou-se principalmente o coeficiente de performance e os resultados de Efeito Frigorífico para cada distribuição de temperatura nas simulações, variando-se a temperatura de condensação e de evaporação respectivamente de, -35 a $-05^{\circ} \mathrm{C}$ e 05 a $35^{\circ} \mathrm{C}$. Em todas as simulações o refrigerante $\mathrm{R} 11$ teve os melhores resultados se tratando de desempenho, mas como o mesmo deixou de ser comercializado em 1996, aplicou-se um comparativo entre os fluidos comercializados, o R22, R134A e R410A. Foi feito também uma análise de custo para os fluidos R22, R134A e R410A e como resultado, o menor custo de trabalho utilizando os dados referentes ao estudo foi obtido com o R22, chegando a R\$ 0,026 .
\end{abstract}

\section{INTRODUÇÃO}

Os processos de refrigeração são utilizados pela humanidade há muitos anos, apenas com variações decorrentes da não utilização da energia elétrica como fonte energética e dos meios refrigerantes, que constantemente estão evoluindo para melhor atender aos processos e às legislações.

Atualmente, e mais ainda em um futuro não tão distante, processos de condicionamento de ar serão muito importantes para a conservação de produtos e processos, indispensáveis para a vida da humanidade, levando em conta o contínuo aquecimento do planeta (Ferraz, 2008).

Por se tratar de sistemas mais complexos para estudos, poucos profissionais buscam a área de refrigeração para especialização, sendo este um dos fatores que podem influenciar uma falta de profissionais no futuro em uma área que, certamente será indispensável para que a população tenha uma qualidade de vida melhor (Ferraz, 2008).

Os objetivos traçados para a realização do presente estudo foram, primeiramente analisar o funcionamento de um refrigerador convencional e estudar os fluidos refrigerantes mais usuais para aplicação em sistemas térmicos e com base nestes, aplicar conhecimentos em programação para realizar uma simulação numérica no ciclo de refrigeração por compressão 
de vapor e chegar em um programa de simulação numérica interativo, que permita ao usuário realizar algumas mudanças em alguns parâmetros para se obter resultados idealizados dos ciclos estudados.

\section{FUNDAMENTAÇÃO TEÓRICA}

Um programa de simulação numérica sempre tem como base, as propriedades específicas dos materiais envolvidos em um determinado processo, as equações e considerações que regem estados ou transformações no espaço e de conhecimento em programação lógica do software que será utilizado para se chegar a tal programa. Tendo conhecimento disso, neste capítulo foram abordados assuntos pertinentes ao: ciclo específico realizado por refrigeradores, freezers e aparelhos de condicionamento de ar e fluidos refrigerantes utilizados atualmente nos ciclos de refrigeração.

\subsection{Ciclo Teórico De Refrigeração Por Compressão De Vapor}

Qualquer ciclo térmico real deveria ter como comparativo o ciclo de vapor de Carnot, por ser este o ciclo de maior rendimento térmico comparado aos demais em condições similares de trabalho. Mas, dado as peculiaridades do ciclo de refrigeração definiu-se um outro ciclo ideal em que, o ciclo real mais se aproxima, e portanto, torna-se mais fácil comparar o ciclo real com este ciclo ideal. Este ciclo ideal é aquele que terá melhor eficácia operando nas mesmas condições do ciclo real, chamado de ciclo teórico simples de refrigeração por compressão.

\section{MATERIAIS E MÉTODOS}

No desenvolvimento da pesquisa foram escolhidos seis fluidos refrigerantes, o R11, o R12, o R22, o R134A, o R410A e o R717. Atualmente o R11 e o R12 não são comercializáveis pelas suas características físico-químicas e o R717 tem muitas restrições no seu comercio, sendo o R22, o R134A e o R410A os fluidos com uma maior comercialização e maior acessibilidade, não tendo restrições para comércio. Neste capítulo foi realizada uma análise mais detalhada desses fluidos, além de caracterizar o software utilizado para realizar as simulações e o programa didático, o Engineering Equation Solver.

\subsection{Fluidos Refrigerantes R22, R134A e R410A}

O fluido R22 é um HCFC, ou seja, um composto de átomos de carbono, hidrogênio, cloro e flúor. Sua vida útil na atmosfera varia entre 2 a 22 anos e, por isso, seu Potencial de Destruição do Ozônio (ODP-Ozone Depletion Potencial) varia de 0,02 a 0,1. Os HCFCs têm sido usados desde o início dos anos 90 e começaram a ser restringidos a partir de 2004 e o R22 deixará de ser comercializado em 2030 (Corrêa, 2010).

Os fluidos R134A e o R410A são compostos de átomos de carbono, hidrogênio e flúor. Por não possuir átomos de cloro, não são nocivos a camada de ozônio e por este motivo, devem ser os refrigerantes mais utilizados em sistemas de condicionamento de ar nas próximas décadas (Corrêa, 2010).

Realizando um comparativo na questão ambiental entre os três fluidos, usando como 
base os valores característicos de ODP e Potencial de Atenção Global (GWP-Global Warning Potencial), o fluido R22 tem respectivamente, valores de 0,05 e 0,34 , já os fluidos R134A e R410A tem ODP 0, e possuem GWP de 0,26 para o R134A e 0,45 para o R410A. Sendo assim, o fluido com as melhores características ambientais é o R134A, por não agredir a camada de ozônio e contribuir menos para os efeitos do aquecimento global.

\subsection{Engineering Equation Solver}

Para a realização das simulações utilizou-se o Engineering Equation Solver (EES), pois o mesmo possui uma vasta biblioteca de equações e fluidos refrigerantes, que foram utilizados nas simulações e também permitem ao usuário, a plotagem dos resultados em uma forma gráfica, através dos gráficos de Mollier, gráfico característico dos fluidos refrigerantes.

\section{RESULTADOS E DISCUSSÕES}

Aplicando conhecimentos obtidos durante o desenvolvimento da pesquisa, foram obtidos resultados quanto a simulação diretamente aplicada a um refrigerador Consul, modelo e ano de fabricação desconhecidos por se tratar de um equipamento antigo e reformado, dele gerou-se simulações aplicando os seis fluidos em estudo e finalmente, chegou-se a um programa didático. Neste capítulo foi abordado sobre o estudo de caso refrigerador Consul e sobre o programa didático.

\subsection{Estudo De Caso Com O Refrigerador Consul}

Usando o Engineering Equation Solver calculou-se o Coeficiente de Performance (COP), a capacidade frigorífica $\left({ }^{Q_{o}}\right)$, o efeito frigorífico $(\mathrm{EF})$, o fluxo de calor rejeitado pelo condensador $\left(\dot{Q}_{c}\right)$ e a vazão mássica de refrigerante $\left(\dot{m}_{f}\right)$ para os fluidos refrigerantes R11, R12, R22, R134A, R410A e R717 (Amônia).

Escolheu-se esses seis fluidos de maneira aleatória, mas levando em conta que o R11 é utilizado como base comparativa para os demais refrigerantes nos índices comparativos de ODP e GWP seria fundamental incluí-lo nesta lista, o R717 e o R12 por serem alguns dos fluidos mais utilizados na história da refrigeração e também, por serem os primeiros a serem utilizados substituindo a água e outros líquidos. O R22 é o fluido mais utilizado atualmente nos sistemas de refrigeração e o R134A e R410A podem ser seus substitutos no futuro, já que o R22 vai sair de linha em 2030 (Corrêa, 2010).

Nota-se na Tabela 1 que o fluido com o maior COP é o R11, consequentemente, possui a maior capacidade frigorífica $\left(\dot{Q}_{o}\right)$ e o maior fluxo de calor rejeitado pelo condensador $\left(\dot{Q}_{c}\right)$. O maior efeito frigorífico (EF) calculado foi o R717, isso deve-se ao fato de que a área abaixo das curvas de saturação abrange uma grande área de entalpia, tendo amplitudes de entalpia maiores que os demais fluidos. Usou-se para esta simulação, temperaturas de evaporação e condensação, respectivamente, $-25^{\circ} \mathrm{C}$ e $35^{\circ} \mathrm{C}$ e potência do compressor $\left({ }^{W}\right)$ de $100 \mathrm{~W}$, que simulam os parâmetros reais adotados nos refrigeradores existentes em nossas residências.

Tabela 1 - Tabela comparativa de resultados. 


\begin{tabular}{|c|c|c|c|c|c|c|c|c|c|}
\hline Run & $R \$$ & $C O P$ & $\begin{array}{c}\dot{W}_{C} \\
{[\mathrm{~kW}]}\end{array}$ & $\begin{array}{c}\dot{Q}_{O} \\
{[\mathrm{~kW}]}\end{array}$ & $\begin{array}{c}E F \\
{[\mathrm{~kJ} / \mathrm{kg}]}\end{array}$ & $\begin{array}{c}\dot{Q}_{C} \\
{[\mathrm{~kW}]}\end{array}$ & $\begin{array}{l}T_{C} \\
{\left[{ }^{\circ} \mathrm{C}\right]}\end{array}$ & $\begin{array}{c}T_{E} \\
{\left[{ }^{\circ} \mathrm{C}\right]}\end{array}$ & $\begin{array}{c}\dot{m}_{f} \\
{[\mathrm{~kg} / \mathrm{s}]}\end{array}$ \\
\hline 1 & RII & 3,434 & 0,1 & 0,3434 & 146 & 0,4434 & 35 & -25 & 0,002353 \\
\hline 2 & R12 & 3,144 & 0,1 & 0,3144 & 106,9 & 0,4144 & 35 & -25 & 0.00294 \\
\hline 3 & $R 22$ & 3,123 & 0,1 & 0,3123 & 151,5 & 0,4123 & 35 & -25 & 0,002061 \\
\hline 4 & R134A & 3,045 & 0,1 & 0,3045 & 134,4 & 0,4045 & 35 & -25 & 0,002265 \\
\hline 5 & R410A & 2,929 & 0,1 & 0,2929 & 155,9 & 0,3929 & 35 & -25 & 0.001879 \\
\hline 6 & $R 717$ & 3,21 & 0,1 & 0,321 & 1065 & 0,421 & 35 & -25 & 0,0003015 \\
\hline
\end{tabular}

A Tabela 1 mostra que em relação aos COP e a vazão mássica, todos os fluidos apresentam valores muito próximos, principalmente se comparar os fluidos R22, R134A e R410A que estão no mercado, sendo utilizados atualmente.

Como o objeto de estudo direto da pesquisa proposta é realizar um comparativo entre alguns fluidos refrigerantes, visando uma análise financeira envolvendo o ciclo de refrigeração proposto e analisando apenas os fluidos comercializáveis atualmente sem alguma restrição, a Tabela 2 apresenta valores de comercialização dos fluidos R22, R134A e o R410A, todos dentro do grupo dos HCFCs e HFCs, que não prejudicam a camada de ozônio.

Tabela 2 - Preço dos Fluidos Refrigerantes.

\begin{tabular}{c|c|c|c}
\hline Fluido Refrigerante & Preço & Massa & Preço/ Quilograma \\
\hline $\mathrm{R} 22$ & $\mathrm{R} \$ 174,00$ & $13,6 \mathrm{~kg}$ & $\mathrm{R} \$ 12,79$ \\
\hline $\mathrm{R} 134 \mathrm{~A}$ & $\mathrm{R} \$ 286,00$ & $13,6 \mathrm{~kg}$ & $\mathrm{R} \$ 21,03$ \\
\hline $\mathrm{R} 410 \mathrm{~A}$ & $\mathrm{R} \$ 437,00$ & $11,3 \mathrm{~kg}$ & $\mathrm{R} \$ 38,67$ \\
\hline
\end{tabular}

Utilizando os valores de vazão mássica calculados para um ciclo de refrigeração de cada tipo de fluido e, utilizando como base de valores a Tabela 2, foi possível obter os resultados do preço gerado através de um ciclo, esses valores são apresentados na Tabela 3.

Tabela 3 - Tabela Comparativa Preço x Performance.

\begin{tabular}{c|c|c|c}
\hline Fluido Refrigerante & COP & $\dot{m}_{f}$ & Preço por ciclo \\
\hline R22 & 3,123 & $0,002061 \mathrm{~kg} / \mathrm{s}$ & $\mathrm{R} \$ 0,026$ \\
\hline R134A & 3,045 & $0,002265 \mathrm{~kg} / \mathrm{s}$ & $\mathrm{R} \$ 0,048$ \\
\hline R410A & 2,929 & $0,001879 \mathrm{~kg} / \mathrm{s}$ & $\mathrm{R} \$ 0,073$ \\
\hline
\end{tabular}

Nota-se na Tabela 3 que o R22 tem os melhores resultados em relação ao COP e ao custo. Em relação a custo, deve-se levar em consideração que o R134A e o R410A são fluidos novos que irão substituir o R22 que tem algumas vantagens em relação a conservação do planeta, o que leva a ter um diferencial em custo, por se tratar de um produto que não agride o meio ambiente.

\subsection{Programa Didático}

Com o procedimento de cálculo já estruturado, propôs-se fazer um programa didático para que se possa realizar iterações quanto à temperatura de condensação, temperatura de evaporação, fluido refrigerante e potência do compressor. Como resultados através desses dados de entrada, calcula-se o COP, o efeito frigorífico, a capacidade frigorífica, o calor 
rejeitado pelo condensador e a vazão mássica do refrigerante. Também, obtêm-se gráficos Pressão-entalpia para os seis tipos de refrigerantes utilizados no programa, sendo os mesmos atualizados a cada alteração realizada no programa.

A Figura 1 mostra a interface que o usuário utiliza para realizar os cálculos, na parte superior é apresentado um esquema simplificado do ciclo de refrigeração por compressão de vapor, mostrando os principais componentes do sistema: o evaporador, o compressor, o condensador e o dispositivo de expansão.

Figura 1 - Interface Programa Didático.

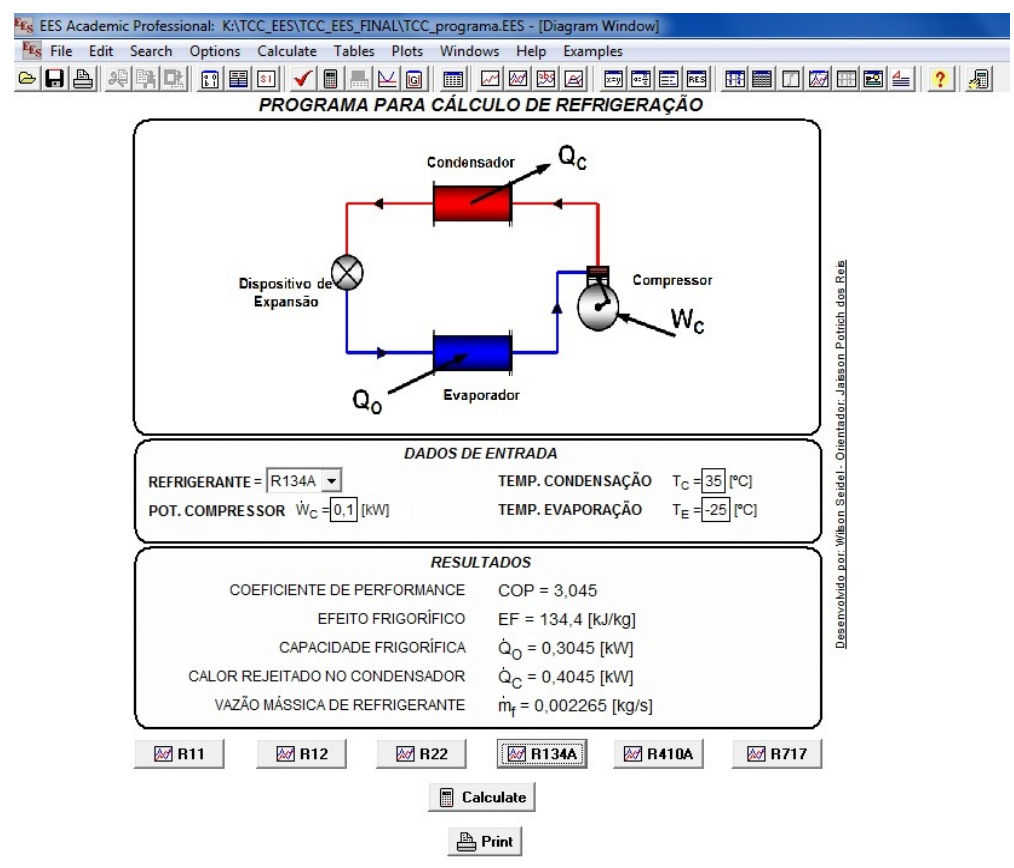

A interface acima apresenta uma simulação que utiliza o fluido refrigerante R134A, com $0,1 \mathrm{~kW}$ de potência do compressor e temperaturas de condensação e evaporação respectivamente, $35 \mathrm{e}-25^{\circ} \mathrm{C}$.

\section{CONSIDERAÇÕES FINAIS}

Os objetivos propostos foram alcançados com êxito, tendo o fluido refrigerante R11 obtido em todas as simulações, os melhores resultados de coeficiente de desempenho. Sabendo que o mesmo não pode ser utilizado nos sistemas de refrigeração devido ao seu alto índice de destruição da camada de ozônio e, realizando um comparativo apenas entre os fluidos disponíveis no mercado, o fluido com o melhor desempenho analisado no estudo foi o R22 que, poderá ser comercializado até 2030, segundo a Conferência de Copenhague (2009). O retrofit do R22 é o R134A, fluido esse que teve desempenho pouco inferior ao R22, mas que pode substituir o mesmo com muita eficácia.

Comparando-se financeiramente os três fluidos, o R22 tem o menor custo de trabalho, utilizando a vazão mássica do mesmo comparado ao seu valor por unidade de massa, tendo custo de R\$ 0,026, o fluido R134A teve custo de R $\$ 0,048$ e o R410A teve custo de R 0,073 . O fluido R22 tem custo de trabalho $84,61 \%$ menor em relação ao fluido R134A e 180,77\% 


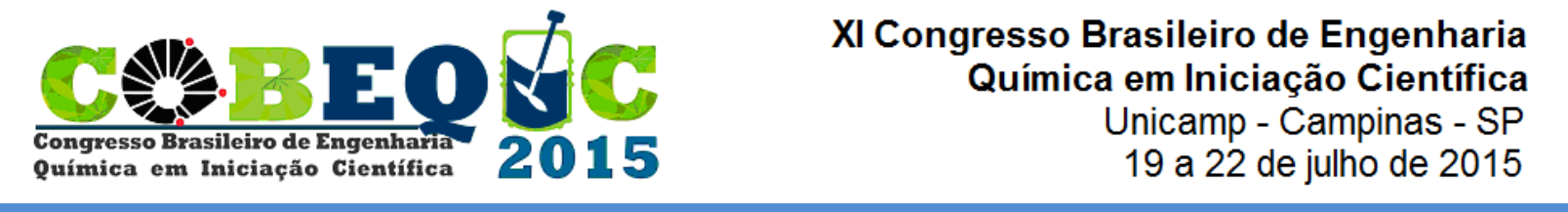

menor em relação ao fluido R410A.

Realizando um comparativo nas questões ambientais entre os três fluidos comercias, o R134A é o menos agressivo, pois não agride a camada de ozônio e contribui menos para os efeitos do aquecimento global, já que não possui o cloro em sua composição química.

Os resultados obtidos com essa pesquisa não são apenas resultados numéricos, podem estar também presentes dentro da sala de aula. O programa didático gerado no decorrer da pesquisa pode ser utilizado em disciplinas de Termodinâmica e Máquinas Térmicas, refrigeração e Ar Condicionado para apresentar os ciclos de refrigeração e mostrar aos acadêmicos as mudanças ocorridas nos processos internos do ciclo refrigerativo e seus resultados diretos, mostrando a variação ocorrida nos resultados com pequenas alterações realizadas nas entradas do programa.

\section{REFERÊNCIAS}

CAMPOS, R. A. S.; SOUZA JR, A. A. A.. Refrigeração e Climatização. Belém, PA. 2009. IFPA - Instituto Federal De Educação, Ciência E Tecnologia Do Pará.

ÇENGEL, Y. A.; BOLES, M. A.. Termodinâmica. 5a edição. São Paulo: Mcgraw-Hill, 2006.

CORRÊA, J. E.. Apostila de refrigeração e Climatização. 2010. UNIVERSIDADE FEDERAL DO PARÁ. Faculdade de Engenharia Mecânica.

DOSSAT, R. J.. Princípios da Refrigeração. Ed. Hemus, 2004.

FERRAZ, F.; GOMES, M.. O histórico da refrigeração, fluidos refrigerantes, ozônio/processo de formação/ destruição, sistemas de refrigeração, componentes de um sistema de refrigeração. 2008. Apostila da disciplina de Refrigeração (Eletromecânica)CENTRO FEDERAL DE EDUCAÇÃO TECNOLOGICA DA BAHIA.

MARTINELli JÚNIOR, L. C.. Refrigeração. Pinambi, RS. 2003. Apostila da disciplina de refrigeração-UNIJUÍ-Universidade Regional do Noroeste do Estado do Rio Grande do Sul. 